\title{
The Generalization and Proof of "Square Root of 2 Is Not a Rational Number" on the Integral Domain
}

\section{Tongchao Liu}

Math Teaching and Research Office in Chengdu College of Arts and Sciences, Chengdu, China

Email: 1223676975@qq.com

How to cite this paper: Liu, T.C. (2021) The Generalization and Proof of "Square Root of 2 Is Not a Rational Number" on the Integral Domain. Advances in Pure Mathematics, 11, 665-669.

https://doi.org/10.4236/apm.2021.117044

Received: June 8, 2021

Accepted: July 5, 2021

Published: July 8, 2021

Copyright ( 2021 by author(s) and Scientific Research Publishing Inc. This work is licensed under the Creative Commons Attribution International License (CC BY 4.0).

http://creativecommons.org/licenses/by/4.0/

\section{(c) (i) Open Access}

\begin{abstract}
In this paper, the traditional proof of "square root of 2 is not a rational number" has been reviewed, and then the theory has been generalized to "if $n$ is not a square, square root of $n$ is not a rational number". And then some conceptions of ring, integral domain, ideal, quotient ring in Advanced algebra, have been introduced. Integers can be regarded as an integral domain, the rational numbers can be regard as a fractional domain. Evens and odds are principal ideals in integral domain. The operations on evens and odds are operations on quotient ring. After introducing "the minimalist form" in fraction ring. The paper proves the main conclusion: in a integral domain, multiplicative subset $S$ produces a fraction ring $S^{-1} R$, and $n$ is not a square element in $R$, then to every element $a \in R, a^{2} \neq n$.
\end{abstract}

\section{Keywords}

Rational Number, Integral Domain, Ideal, Fraction Ring, Generalize

\section{Introduction}

$\sqrt{2}$ is an irrational number, which is an indisputable fact, was proved by an ancient Greek mathematician Hippasus though a method named contradiction. The proof is shown as below: if $\sqrt{2}$ is a rational number $\frac{p}{q}$ ( $p, q$ are co-prime integers), and then $p^{2}=2 q^{2}$, then $p^{2}$ is an even. By the theory: the square of an odd number is an odd, and so is an even number, we can draw a conclusion that $p$ is an even. Assume that $p=2 k$, then $p^{2}=4 k^{2}$ is divisible by 4 , then $q^{2}$ is an even, then $q$ is an even. So $p, q$ are both even, which is contradict by the premise: $p, q$ are co-prime integers. 
Two implications can be got from the classical proof.

1) In the proof, all the integers are divided into two equivalence classes: odds and evens, the two equivalence classes are closed to the "square" operation. And by the method "contradiction", $p$ and $q$ are in a same class: evens, which is a contradiction. What's more, for a general positive integer $n$, which is not a perfectly squared, is its square root an irrational? Whether it can also be proved by constructing an equivalence class?

2) The set of integers form a ring by ordinary addition and ordinary multiplication, and the ring of rational numbers is a fractional ring generated by the ring of integers. "The square root of 2 is not a rational number" can be extended to ordinary ring? If not, can we make the conclusion correctly by strict the ring; if so, how the conclusion could be proved?

When it comes to how to find the square root of an element in a ring, a Chinese researcher named Miaoqin Chen [1] found a way to solve the problem in Rings of polynomials with rational coefficients. But to a general integral domain, few researchers solve the problem.

David E. Dobbs [2] did some researches about why the square root function is not linear in a ring.

\section{2. $n$ Is Not a Perfect Square Number, and the Square Root of Which Is an Irrational}

Define 2.1. $n$ is a positive integer, $[a]_{n}=\{r+q n \mid q$ is an intenter $\}(r=0,1,2, \cdots)$ is a residue class module $n$. The set of all the residue classes denote as $Z_{n}$.

It can be known easily that $Z_{n}$ forms a partition of the set of integers. Every integer belongs and only belongs one class of $Z_{n}$, Thereafter, in the case of ambiguity, $[a]_{n}$ can be denoted as $[a]$.

Lemma 2.1. If $n$ is not a perfect square integer $\forall k(1 \leq k<n), k^{2} \neq n, b$ is a positive integer, if $b \in[0]$, then $b^{2} \in[0]$, if $b \notin[0]$, then $b^{2} \notin[0]$

Proof: assume that $b=r+q n(0 \leq r<n)$, then $b^{2}=r^{2}+2 r q n+q^{2} n^{2} \in\left[r^{2}\right]$,

Case 1: when $b \in[0]$, it means that $r=0$ and $b^{2} \in[0]$.

Case 2: when $b \notin[0]$, because $r^{2} \neq n$, and $b^{2} \notin[0]$.

From the proof above, the conclusion below can be easily proved: to any positive integer $m$, if $b \in[0]$, then $b^{m} \in[0]$, if $b \notin[0]$, then $b^{m} \notin[0]$.

Lemma 2.2. Assuming that $b, n$ are both positive integers, then $b \in[0]_{n} \Leftrightarrow n b \in[0]_{n^{2}}$.

Proof: $b \in[0]_{n} \Leftrightarrow b=q n, q \in Z, \quad n b \in[0]_{n^{2}} \Leftrightarrow n b=p n^{2}, q \in Z, \quad p \in Z$, let $p$ $=q$, and the conclusion is obviously right.

Theorem 2.1. If $n$ is not a perfect square integer, $\sqrt{n}$ is an irrational number.

Proof by contradiction: if $\sqrt{n}$ is a rational number, let us assuming that it is $\sqrt{n}=\frac{p}{q}$, in which $p$ and $q$ are co-prime, and $p^{2}=n q^{2}$, based on the definition of the residue classes of module $n, p^{2} \in[0]_{n}$. Because of the Lemma $2.1 \quad p \in[0]_{n}$, 
we know that $p^{2} \in[0]_{n^{2}}$ by lemma 2.2, and Because of the lemma 2.1, $q \in[0]_{n}$, so $p$ and $q$ have the same factor $n$, which is incompatible to the premise: $p$ and $q$ are co-prime.

As the procedure mentioned above, the theorem can be extended as: if $n$ is not a m power number (to any positive integer $k k(1 \leq k<n), k^{m} \neq n$ ), then $\sqrt[m]{n}$ is an irrational number, the proof will not be shown in the essay.

\section{Some Important Conceptions and Conclusions in Ring Theorem}

To extend "the square root of 2 is not a rational number" to a general ring, some concepts about ring, ideal will be used, and some new methods, like minimalist, will be invented. So let's review some definitions and theorems of abstract algebra.

Definition 3.1. [3] Assume that $R$ is a non-empty set. If we define two algebraic operation on $R$, one is called addition, noted as $a \oplus b$, and the other one is called multiplication, noted as $a \otimes b$, and they meet the following conditions:

1) $R$ form a Abelian group on the operation $\oplus$.

2) The associative law of multiplication: to and elements $a, b, c$, it meet $(a \otimes b) \otimes c=a \otimes(b \otimes c)$.

3) The distributive property of multiplication over addition. To any elements $a, b, c \in R$, they meet:

$$
\begin{aligned}
& c \otimes(a \oplus b)=(c \otimes a) \oplus(c \otimes b) \\
& (a \oplus b) \otimes c=(a \otimes c) \oplus(b \otimes c)
\end{aligned}
$$

Then $R$ is a ring.

If the multiplication in $R$ also meets the commutative law, $R$ is said to be a commutative ring.

If there is an element $e$ fit the condition: to any element $a$ in $R, a \otimes e=e \otimes a$, then $e$ is called an identity.

A commutative ring without zero divisor but with a identity is called a integral domain, which is a mainly study object in this essay.

Example: $n$ is a positive integer, over the operations $[a] \otimes[b]=[a \times b]$,

$[a] \oplus[b]=[a+b]$ is a commutative ring, and the identity is $e=[1]$, if $n$ is a prime, $Z_{n}$ is a integral domain.

Example: integer set $Z$ over the normal addition and multiplication form a ring, called integer domain.

Definition 3.2. [4] [5] Let $R$ be a integral domain, a subset $R$ of $S$ is called a multiplicative closed subset, if the identity $e \in S$, zero $O \notin S$, and $S$ is closed over multiplication, it means $\forall s_{1} \in S, s_{2} \in S, s_{1} \otimes s_{2} \in S$

Definition 3.3. [6] [7] Let $R$ be a integral domain, $S$ is a multiplicative closed set, a equivalence relation " $\sim$ " can be defined in the set $R \times S$, $\left(r_{1}, s_{1}\right) \sim\left(r_{2}, s_{2}\right) \Leftrightarrow r_{1} \otimes s_{2}-s_{2} \otimes r_{1}=O$

Note $S^{-1} R=R \times S / \sim$ is a set produced by $S^{-1} R$ in the relative of $R \times S$, 
we use $\frac{r}{s}$ to represent the equivalence class produced by $(r, s)$.

Addition and multiplication can be defined in $S^{-1} R$ :

$$
\begin{gathered}
\frac{r_{1}}{s_{1}} \oplus \frac{r_{2}}{s_{2}}=\frac{\left(r_{1} \otimes s_{2}\right) \oplus\left(r_{2} \otimes s_{1}\right)}{s_{1} \otimes s_{2}} \\
\frac{r_{1}}{s_{1}} \otimes \frac{r_{2}}{s_{2}}=\frac{r_{1} \otimes r_{2}}{s_{1} \otimes s_{2}}
\end{gathered}
$$

So, $S^{-1} R$ is a commutative ring with identity over the addition and multiplication mentioned above. $S^{-1} R$ is called a fractional ring over $S$, because of $e \in S, R$ can be isomorphic embedded in $S^{-1} R$.

Example: To integer domain $R, S=R \backslash\{0\}$, the fractional ring over $S$ is rational number ring $Q$.

Definition 3.4. [7] Let $R$ be a commutative ring with identity, $I$ is a nonempty set of $R$. if $I$ form a sub group over the operation $\oplus$, and I have absorption, that is:

$$
a \in I, r \in R \Rightarrow a \otimes r \in I
$$

Then $I$ is called an ideal.

Example: $a \in R,(a)=\{r \otimes a \mid r \in R\}$ is an ideal of $R$, the ideal is called a principal ideal. If $(a)=O$ or $(a)=R$, the ideal is called a trivial ideal, or it is called Nontrivial ideal.

Example: in integer number domain $Z$, the set of evens $2 Z$ forms an ideal.

Definition 3.5. The cosets $r+I$ of ideal $I$ of a ring $R$ form a division of $R$, over the addition operation $\left(r_{1}+I\right) \oplus\left(r_{2}+I\right)=r_{1} \oplus r_{2}+I$ and the multiplication $\left(r_{1}+I\right) \otimes\left(r_{2}+I\right)=r_{1} \otimes r_{2}+I$, the cosets forms a domain, it is called quotient ring.

\section{The Extension and Proof in a Ring of "The Square Root of a Non-Square Number Is Not Rational Number"}

Theorem 4.1. Let $R$ be an integral domain, $n \in R, n$ is a not-square number ( $\forall r \in R, r \otimes r \neq n$ ), $S$ is a multiplication closed set, $S^{-1} R$ is a fractional ring of $R$ over $s$, then, to any $a \in S^{-1} R, a^{2}=a \otimes a \neq n$.

Before theorem 4.1 is proved, some definitions and lemmas should be introduced.

Definition 4.1. In a fractional ring $S^{-1} R$ of $R$ over $S$, the minimalist style of the element $\frac{r}{s}$ is $\frac{r_{1}}{s_{1}}$, if $\frac{r}{s} \sim \frac{r_{1}}{s_{1}}$, and $r_{1}, s_{1}$ not belong to any nontrivial principal ideal.

Lemma 4.1. Let $R$ is an integral domain, every element in $S^{-1} R$ has a minimalist style.

Proof: Assume that $\frac{r}{S} \in \frac{R}{S}$, if $r, s$ both belong to a principal ideal, that is $r, s \in(a), a \neq e$, then $r=a \otimes r_{1}, \quad s=a \otimes r_{2}$, and 


$$
\frac{r}{s}=\frac{a \otimes r_{1}}{a \otimes s_{1}} \sim \frac{r_{1}}{s_{1}}\left(\because a \otimes r_{1} \otimes s_{1}-a \otimes s_{1} \otimes r_{1}=O\right)
$$

Lemma 4.2. Let $R$ be an integral domain, $n$ is a non-square element in $R$, then $a \in(n) \Leftrightarrow a^{2} \in(n)$.

Proof: “ $\Rightarrow$ ": $a \in(n) \Rightarrow \exists r \in R, a=n \otimes r \Rightarrow a^{2}=n \otimes(r \otimes n \otimes r) \Rightarrow a^{2} \in(n)$

" $\Leftarrow$ ": because of the definition of quotient ring $R /(n)=\{r+(n) \mid r \in R\}$, $R /(n)$ form a division of $R$, so $\exists s \in R, a \in s+(n)$, so, $a^{2} \in s^{2}+(n)$, if $a^{2} \in(n)$, then $s^{2} \in(n)$, because $n$ is not a perfect square element, so $s^{2} \neq n$, so $s^{2}=O$, because $\mathrm{R}$ is an integral domain, so $s=O$, so $a \in(n)$.

The proof of theorem 4.1 is shown as below:

Proof: if there is an element $a \in S^{-1} R$, meet $a^{2}=a \otimes a=n$, and the minimalist style of a is $\frac{r}{s}$ (which means $r$ and $s$ are not in any nontrivial ideal), then $r^{2}=s^{2} \otimes n, r^{2} \in(n)$, because of lemma 4.2, $r \in(n), \quad r \in(n)$, then $r^{2} \in\left(n^{2}\right)$. Assume that $r^{2}=k \otimes n^{2}=s^{2} \otimes n(k \in R)$, so based on the elimination law, we can get that $s^{2}=k \otimes n$, which means $s^{2} \in(n)$, based on the lemma 4.2, $s \in(n)$, because $r \in(n)$ and $r \in(n)$ and $(n)$ is a nontrivial principal ideal, it is paradox to $\frac{r}{S}$ a minimalist style.

So, to any $a \in S^{-1} R \quad a^{2}=a \otimes a \neq n$.

But what is worth for emphasizing is that sometimes it is not easy to judge if an element is a square element, for example, in the 2 order matrix ring generated by $Z, \quad 2=e \oplus e=\left[\begin{array}{ll}2 & 0 \\ 0 & 2\end{array}\right]=\left[\begin{array}{cc}1 & 1 \\ 1 & -1\end{array}\right]^{2}$, so 2 is not a square element.

\section{Conflicts of Interest}

The author declares no conflicts of interest regarding the publication of this paper.

\section{References}

[1] Chen, M.Q. (2009) Square Root Algorithm of Elements in Quotient Ring of Polynomial Ring with Rational Coefficients. Journal of Yibin University. (in Chinese)

[2] Dobbs, D.E. (2002) Why the Square Root Function Is Not Linear. International Journal of Mathematical Education in Science and Technology, 33, 742-747. https://doi.org/10.1080/002073902320602897

[3] Qiu, W.S. (2015) Foundation of Abstract Algebra. Higher Education Press, Beijing. (in Chinese)

[4] Tang, Z.M. (2015) Introduction to Commutative Algebra. Science Press, Beijing. (in Chinese)

[5] Wang, W.L. and Ren, Y.L. (2012) Fractional Domains of General Domains. Mathematical Theory and Applications, 1, 81-84. (in Chinese)

[6] Picozza, G. and Tartarone, F. (2010) Flat Ideals and Stability in Integral Domains. Journal of Algebra, 324, 1790-1802. https://doi.org/10.1016/j.jalgebra.2010.07.021

[7] Shi, S.M. (2006) Preliminary Study of Modern Algebra. Higher Education Press, Beijing, 5-7. (in Chinese) 